\title{
Revascularização miocárdica por minitoracotomia esquerda: série de casos
}

\author{
Left mini-thoracotomy off-pump coronary revascularization
}

Theófilo GAUZE ${ }^{1}$, Flávio de Almeida ROSA², Waldir Ferreira de SALVI JR ${ }^{3}$, Elzio TAMAZATO4

RBCCV 44205-876

\section{Resumo}

Objetivo: A reestenose é uma das complicações freqüentes das angioplastias. Estudos demonstraram superioridade da anastomose de Artéria Torácica Interna Esquerda (ATIE) para Coronária Interventricular Anterior (DA). Discute-se a indicação, técnica operatória e resultados da Revascularização do Miocárdio por Minitoracotomia Esquerda (MTE) sem extracorpórea.

Método: Foram operados 18 pacientes (três mulheres), idade de 56,6 $\pm 9,2$ anos, com "shunt” intracoronário, para anastomose da ATIE para DA (14 casos) ou Diagonal (DI) e DA (4 homens). Exposição e estabilização foram obtidas com dispositivo Access (CardioThoracicSystems ${ }^{\circledR}$ ). $O$ enxerto foi dissecado esqueletizado e anastomosado com fio único de polipropileno 7-0.

Resultados: Não ocorreu óbito, conversão para esternotomia, transfusões ou alterações enzimáticas. As altas hospitalares ocorreram entre 3 e 5 dias e todos os pacientes retomaram suas atividades em até 20 dias. Houve uma reinternação por oclusão do enxerto, tratada com angioplastia, e outra por infeç̧ão incisional. Seis pacientes reestudados voluntariamente apresentaram angiografia com enxertos pérvios.

Conclusão: A abordagem mostrou-se segura e sem obstáculos técnicos. Houve tempo de hospitalização curto e baixa morbidade, sem uso de hemoderivados. $O$ instrumental apropriado e, casualmente, a aptidão manual esquerda do cirurgião facilitaram a técnica. Estudos randomizados poderão demonstrar se há benefício econômico e/ou clínico, no longo prazo, desta abordagem como tratamento preferencial.

Descritores: Revascularização miocárdica, métodos. Procedimentos cirúrgicos minimamente invasivos. Artéria torácica interna. Tórax, cirurgia.

\footnotetext{
1. Membro Titular da SBCCV;Cirurgião Cardiovascular e Torácico

2. Especialização, Cirurgião Assistente do Serviço.

3. Especialista SBC / Ecocardiografista, Cardiologista do Pósoperatório.

4. Especialista em Cardiologia / SBC, Coordenador do Pós-operatório.

Trabalho realizado na Clínica Cárdio-Cirúrgica Dr.Theófilo Gauze Santa Casa de Campo Grande, MS.
}

\section{Endereço para correspondência:}

Dr.Theófilo Gauze. Rua Maracá, 325 - Vila Rica - Campo Grande MS - CEP 79022-270 Tel: (67) 3383-6598 / 9981-7816.

E-mail: tgauze@cardiol.br 


\begin{abstract}
Objectives: Stent restenosis is a common complication in angioplasty. Studies have shown better outcomes when the left internal thoracic artery (LITA) is anastomosed to the left anterior descending artery (LAD). Patient selection, operative technique and results for off-pump left mini-thoracotomy (LME) coronary surgery, as a pilot study, are presented.

Methods: Eighteen patients (three women) with a mean age of 56.6 \pm 9.2 were operated on through a 9-14 cm LME to perform off-pump LITA to LAD anastomoses (14 patients) or diagonalis (DI) and LAD sequential anastomoses (4 men). The grafts were skeletonized during dissection and anastomoses were performed using 7-0 polypropylene running sutures. An access device (CardioThoracic Systems $\left.{ }^{\circledR}\right)$ allowed approach and coronary stabilization.

Results: There were no deaths, conversion to sternotomy, transfusions or high enzyme levels. All patients were released
\end{abstract}

\section{INTRODUÇÃO}

O fenômeno de reestenose tem sido um dos problemas freqüentes e indesejáveis nas dilatações arteriais por cateterbalão, seguidas do implante de próteses mecânicas de suporte intraluminar ou "stents" [1]. O advento das próteses recobertas com fármacos melhorou o resultado destes procedimentos, entretanto, a presença de reestenoses intraprótese persiste, ainda que em menor incidência [2-4]. Estudos de longo prazo demonstraram que a cirurgia para revascularização do miocárdio (RM) com uso da artéria torácica interna esquerda (ATIE) para a coronária interventricular anterior (DA) produziu mais alívio de sintomas, menor incidência de reintervenção e melhor preservação da fração de ejeção do ventrículo esquerdo, comparativamente ao tratamento clínico medicamentoso e também à angioplastia [5].

O surgimento de novos dispositivos estabilizadores e os “shunts" intracoronarianos, assim como o desenvolvimento de afastadores e técnicas de abordagem cirúrgica por acessos minimamente invasivos, reacendeu o interesse pela avaliação comparativa dos verdadeiros custos e benefícios que envolvem as abordagens disponíveis para a RM - considerando-se agora a evolução de longo prazo.

Em 2001, após preparativos e treinamentos, passamos a realizar a cirurgia para RM sem o suporte da circulação extracorpórea (CEC) e com dispositivos estabilizadores epicárdicos variados. Nesta época, também utilizamos os dispositivos para perfusão intracoronariana como forma de proteção ao miocárdio isquêmico durante a realização das anastomoses distais. Progressivamente, abordamos uma porcentagem cada vez maior de pacientes atingindo hoje próximo de $80 \%$ dos casos de cirurgia para RM com esta tática (comunicação). Com o advento da experiência, começamos a desenvolver um projeto de estudo piloto para a RM através de acesso minimamente invasivo por from hospital on the 3rd to 5 th postoperative days and returned to their day-to-day activities within 30 days. One woman was readmitted for angina, presented with graft occlusion, and a stent was implanted and one man was readmitted for wound infection. Future angiography was performed on six patients and showed patent grafts.

Conclusion: The operation was performed with low morbidmortality, short hospital stays and without transfusions. Appropriate instruments and the ability of the surgeon to use his left hand, made this operation technically easy. Randomized studies may prove if there are sufficient clinical and economic benefits over the long term to make this surgery the first choice.

Descriptors: Myocardial revascularization, methods. Surgical procedures, minimally invasive. Mammary arteries. Thorax, surgery.

toracotomia anterior esquerda (MTE), abordando inicialmente os ramos anteriores da coronária esquerda e sem o suporte da CEC para tratamento das obstruções de coronarianas descendente anterior e diagonal [6-8]

\section{MÉTODO}

No período de janeiro de 2004 a fevereiro de 2006, foram operados 18 pacientes portadores de insuficiência coronariana por meio de cirurgia para RM por MTE. O projeto foi apreciado pela Comissão de Ética da Instituição e todos os pacientes assinaram detalhado Consentimento Esclarecido antes da operação. A idade média foi de 56,6 \pm 9,2 anos, sendo três (16,6\%) pacientes do sexo feminino e 15 (72,2\%) do masculino. Todos os pacientes eram sintomáticos e apresentaram teste de esforço positivo na avaliação pré-operatória. A fração de ejeção do ventrículo esquerdo (Teicholtz) variou de 34 a 68\% (51,2 \pm 8,5\%). Comorbidades apresentaram-se como segue: hipertensão arterial sistêmica em cinco (27,7\%), diabete melito cinco (27,7\%), infarto do miocárdio e angioplastia prévios em quatro (22,2\%), doença pulmonar obstrutiva crônica em seis (33,3\%), vasculopatia periférica importante em dois (11,1\%) e envolvimento de significativo ramo diagonal (DI) em quatro $(22,2 \%)$. O acesso utilizado foi a MTE com incisões que variaram de 9 a $14 \mathrm{~cm}$ de extensão. O dispositivo Access (CardioThoracicSystems ${ }^{\circledR}$ - Figura 1) foi utilizado para exposição da ATIE e sua dissecção na forma esqueletizada; sendo seus ramos ligados com clipes de titânio (Johnson LT200®), na porção proximal, e cauterizados com bisturi eletrônico na porção distal. Após o preparo do enxerto e abertura do pericárdio, avaliou-se a adequada extensão do mesmo. A heparinização consistiu em uma única dose de 1,5 $\mathrm{mg} / \mathrm{kg}$ peso e, simultaneamente, administrou-se nestes pacientes uma dose sublingual de 200mg de ácido acetilsalisílico. Com o afastador e seu sistema de estabilização 
devidamente instalados sobre a artéria coronária DA, procedeu-se à arteriotomia coronariana sem garroteamento do vaso. A hemostasia nesta fase foi proporcionada pela simples compressão proximal da artéria pela porção posterior da pinça anatômica do cirurgião auxiliar. Instalou-se o "shunt" perfusor intracoronariano (Figura 2) e procedeu-se à anastomose direta da ATIE com a DA por sutura contínua com fio de polipropileno 7-0 (Figura 3). Não houve isquemia maior do que 30 segundos em nenhum caso até a instalação do "shunt" e reperfusão. Após revisão da hemostasia sob protamina, instalou-se dreno torácico com diâmetro de $28 \mathrm{~mm}$ sob visão direta e conectou-se ao sistema de drenagem fechada em selo d’água.

\section{RESULTADOS}

Todos os 18 pacientes puderam ser revascularizados com sucesso, sendo que quatro receberam anastomoses

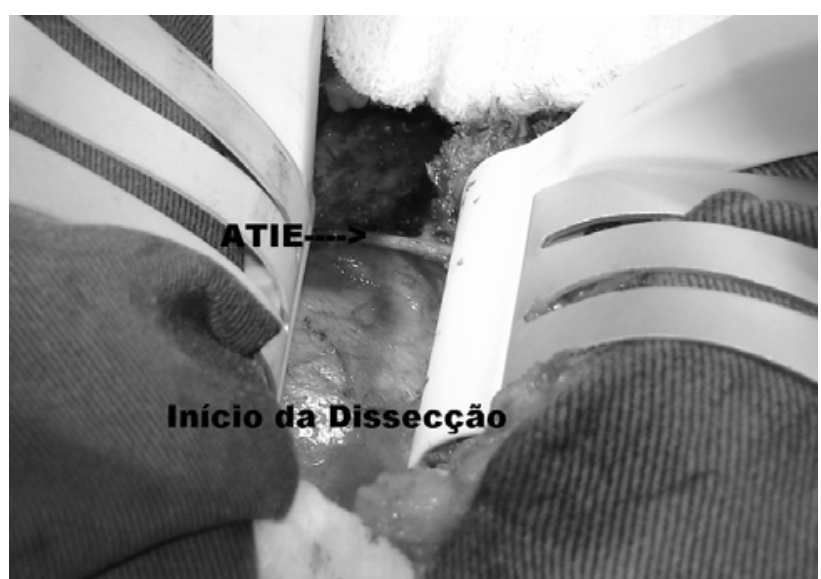

Fig. 1 - Dispositivo afastador para dissecção da ATIE por minitoracotomia

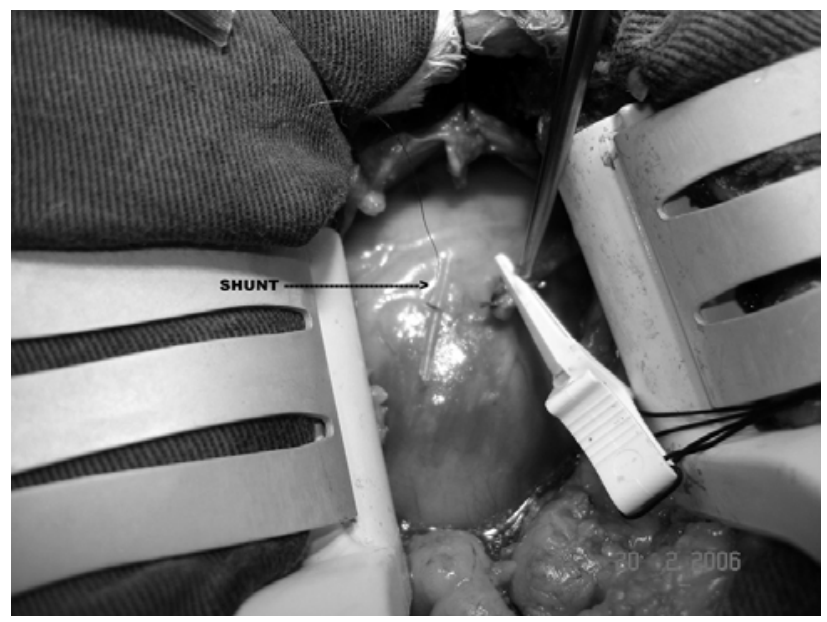

Fig. 2 - Perfusor ("shunt") intracoronariano seqüenciais para coronária DI-DA (1,2 ponte/paciente). Não houve óbitos, transfusões ou alterações enzimáticas e eletrocardiografias. Não foi necessária a conversão para esternotomia. $\mathrm{O}$ tempo de permanência na Unidade Intensiva Cardiológica variou de 18 a 36 horas (24,3 \pm 8,5horas). A evolução clínica pós-operatória (PO) foi adequada e as altas hospitalares puderam ser efetuadas entre o terceiro e o quinto dia PO (mediana 3,5) em todos os pacientes; que retomaram suas atividades rotineiras em até 20 dias. $\mathrm{O}$ resultado estético, em geral, foi satisfatório (Figura 4). Houve uma reinternação ( $34^{\circ}$ dia PO - sexo feminino) por angina estável e oclusão do enxerto à angiografia; esta paciente foi tratada por meio de angioplastia da coronária DA. Ocorreu uma reinternação por infecção incisional ( $6^{\circ}$ dia $\mathrm{PO}$ - sexo masculino) tratada com antibióticos. Seis pacientes foram reestudados voluntariamente e apresentaram enxertos com fluxo normal (Figura 5). À exceção do paciente com oclusão de enxerto, todos os demais tiveram o teste ergométrico negativo após 60 dias da operação.

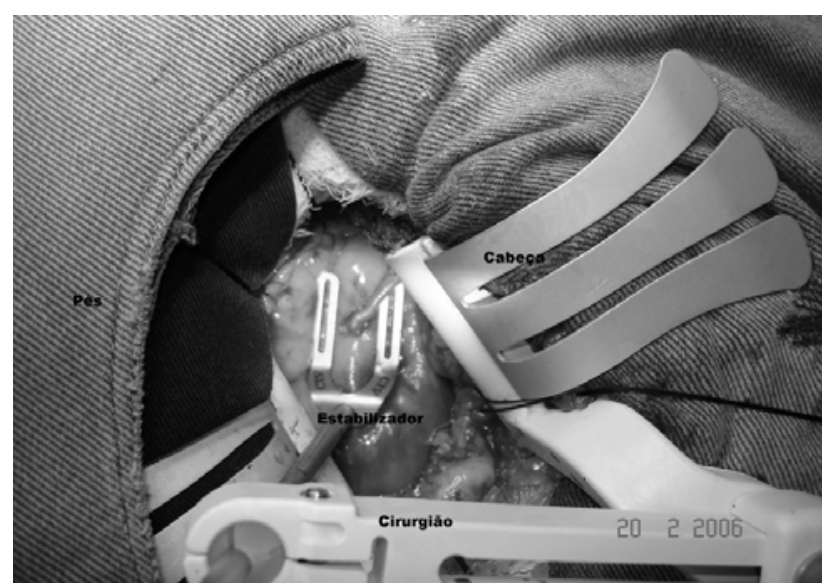

Fig. 3 - Anastomose com fio 7-0 posicionada sob dispositivo estabilizador

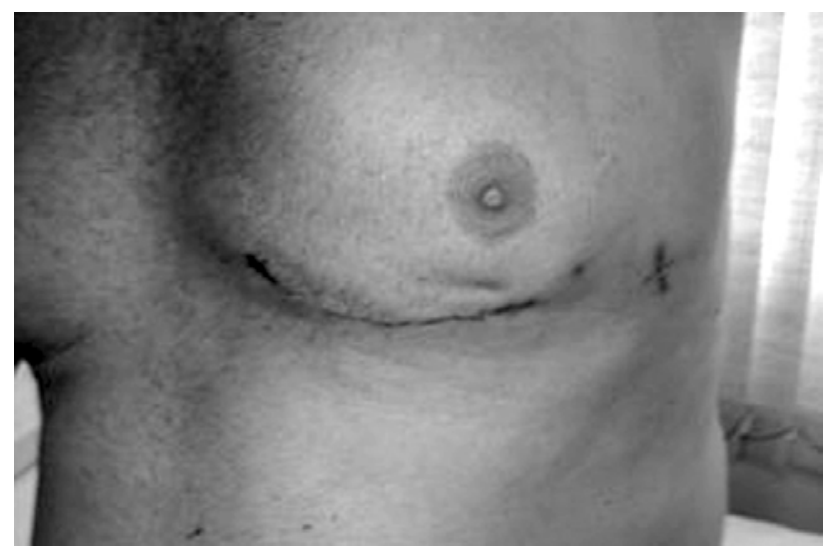

Fig. 4 - Aspecto estético cicatricial 


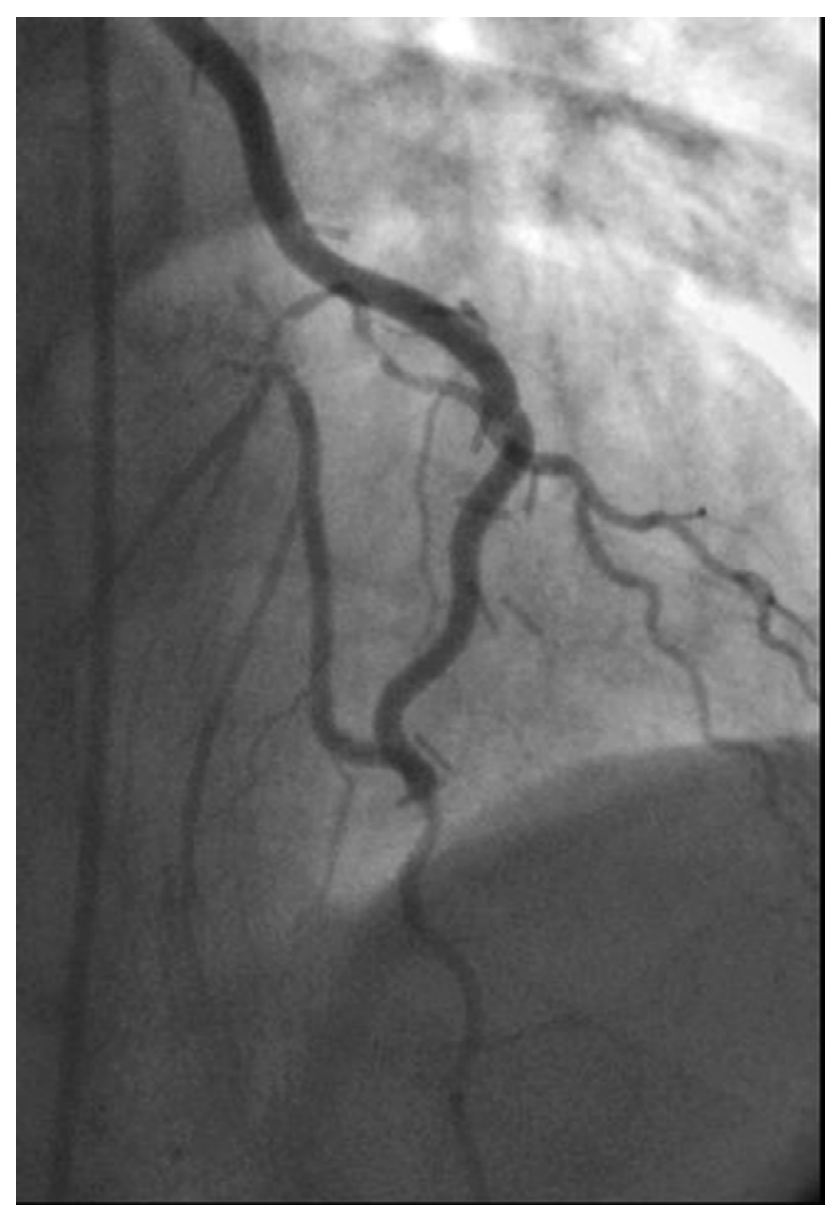

Fig. 5 - Angiografia pós-operatória tardia demonstrando anastomose seqüencial de ATIE para coronárias DI e DA.

\section{DISCUSSÃO}

A abordagem mostrou-se segura e sem obstáculos técnicos. O instrumental apropriado facilitou a técnica, pois permitiu a adequada exposição da ATIE pela elevação dos arcos costais. A ventilação seletiva foi possível em 10 pacientes, nos quais não ocorreu alteração da capnografia e simplificou a dissecção da ATIE. Um elemento casual que surpreendeu foi o fato de que a aptidão manual esquerda (cirurgião canhoto - Figura 6) permitiu o posicionamento confortável dos instrumentos para a dissecção da ATIE até o seu primeiro ramo intercostal. Esse achado permite-nos inferir que o uso da videotoracoscopia poderá facilitar em muito esta abordagem. A esqueletização do enxerto permitiu maior extensão do mesmo, evitando tensão sobre as pontes, mesmo nos casos de anastomoses seqüenciais. $\mathrm{O}$ tempo de hospitalização reduzido e a baixa morbidade, sem uso de hemoderivados, certamente são objetivos interessantes alcançados nesta série.

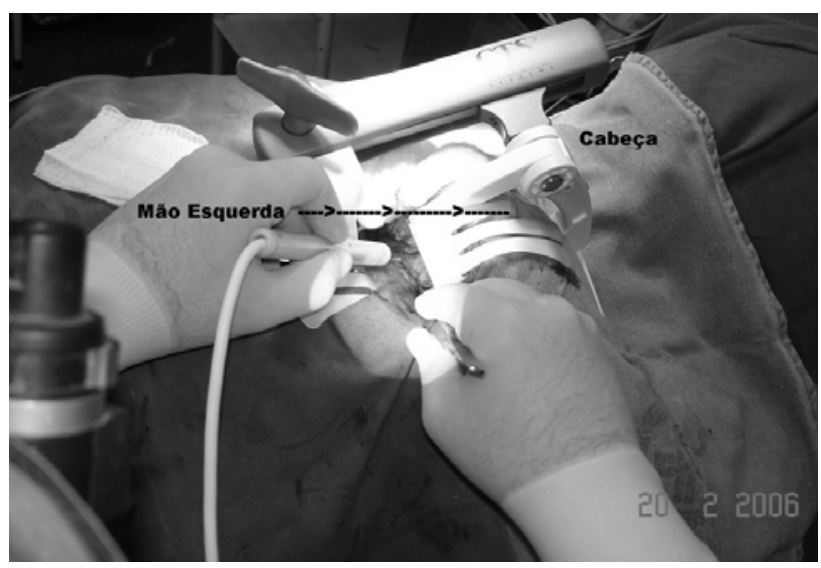

Fig. 6 - Posição ergonomicamente confortável para o cirurgião canhoto

O crescimento das populações de maior risco, como os diabéticos [9] e os pacientes submetidos a múltiplos procedimentos [10], pode mudar a maneira como indicamos a cirurgia para a RM face ao custo adicional de reintervenções com uso das próteses farmacológicas de alto custo.

\section{CONCLUSÕES}

A técnica foi realizada com sucesso em todos os pacientes. Observou-se baixa morbi-mortalidade e os pacientes apreciaram a estética da incisão. Estudos complementares poderão determinar se e quando pode haver benefício desta operação como tratamento preferencial na RM dos vasos da parede anterior do ventrículo esquerdo.

\section{REFERÊNCIAS}

1. Gomes WJ, Buffolo E. Stent coronário e inflamação. Rev Bras Cir Cardiovasc. 2003;18(4):III-VII.

2. Farb A, Sangiorgi G, Carter AJ, Walley VM, Edwards WD, Schwartz RS, et al. Pathology of acute and chronic coronary stenting in humans. Circulation. 1999;99(1):44-52.

3. Taniyasu N, Akiyama K, Hirota J, Iba Y. Newly developed left main coronary artery lesion after coronary stenting. J Cardiovasc Surg. 2002;43(1):55-8. 
4. Katritsis DG, Karvouni E, Ioannidis JP. Meta-analysis comparing drug-eluting stents with bare metal stents. Am J Cardiol. 2005;95(5):640-3.

5. Hueb WA, Bellotti G, Olivera SA, Ariê S, Albuquerque CP, Jatene AD, et al. The Medicine, Angioplasty or Surgery Study (MASS): a prospective, randomized trial of medical therapy, balloon angioplasty or bypass surgery for single proximal left anterior descending artery stenoses. J Am Coll Cardiol. 1995;26(7):1600-5.

6. Alessandrini F, Luciani N, Marchetti C, Guadino M, Possati G. Early results with the minimally invasive thoracotomy for myocardial revascularization. Eur J Cardiothorac Surg. 1997;11(6):1081-5.

7. Schaff HV. New surgical techniques: implications for the cardiac anesthesiologist: mini-thoracotomy for coronary revascularization without cardiopulmonary bypass. J Cardiothorac Vasc Anesth. 1997;11(2 Suppl 1):6-9; discussion 24-5.

8. Biglioli P, Antona C, Alamanni F, Parolari A, Toscano T, Pompilio G, et al. Minimally invasive direct coronary artery bypass grafting: midterm results and quality of life. Ann Thorac Surg 2000;70(1):456-60.

9. Influence of diabetes on 5-year mortality and morbidity in a randomized trial comparing CABG and PTCA in patients with multivessel disease: the Bypass Angioplasty Revascularization Investigation (BARI). Circulation. 1997;96(6):1761-9.

10. Singh SK, Mishra SK, Kumar D, Yadave RD, Sinha SK. Multivessel total arterial revascularization via left thoracotomy. Asian Cardiovasc Thorac Ann 2004;12(1):30-2. 